\title{
DESIGN OF CAN-BASED VARIABLE RATE FERTILIZER CONTROL SYSTEM
}

\author{
Wenqian Huang ${ }^{1, *}$, Zhijun Meng ${ }^{1}$, Liping Chen ${ }^{1}$, Chunjiang Zhao ${ }^{1}$ \\ ${ }^{1}$ National Engineering Research Center for Information Technology in Agriculture, China \\ 100097 \\ * Corresponding author, Address: Room A516, Nongke Building, Shuguang Garden Middle \\ Road, Haidian District, Beijing, 100097, P. R. China, Tel: +86-10-51503425, Fax: +86-10- \\ 51503750, Email: huangwq@nercita.org.cn
}

Abstract: A novel control system for variable rate fertilizer application based on CAN bus is presented. The system consists of variable rate controller based on DSP, ground speed sampling unit and light-bar guidance unit. These units form a control area network based on CAN bus. The variable rate controller collects signals of GPS position and ground speed, and calculates the conveyor feed shaft rotate speed according to the prescription map stored in local memory. Then the variable rate application can be realized through PID control of the shaft rotate speed. Light-bar guidance unit helps the operator to steer the machine moving by equal spacing parallel passes for less overlap and missing in operation. The effectiveness of the developed system is confirmed by the experiments using the communication protocol special for the application based on CAN bus.

Keywords: Precision Agriculture, variable rate fertilizer, CAN bus, PID control, DSP

\section{INTRODUCTION}

In recently years, Precision Agriculture (PA) is becoming one of the most important fields in research of agriculture science. Precision Agriculture is the integrated application of GPS, RS, GIS and automatic control technologies. By knowing the different characteristics in each field cell, maximum profit can be obtained by balancing precise amounts of inputs with crop needs, which is determined by weather, soil characteristics and 
historic crop performance. In addition, precision agriculture techniques will more closely meet environmental guidelines because fertilizers, insecticides, and herbicides can be managed to apply the minimum needed for effectiveness (Wang 1995). As an important part of precision agriculture technology, much attention has been paid to the variable rate technology. Variable rate application has the potential to improve fertilizer utilization efficiency, increase economic returns and reduce environmental impacts. Today, developed countries have used variable rate technology widely, but it is still in the starting stage in China. The rapid development of precision agriculture has increased the need for a standardized electronics communications protocol also (Marvin L. Stone et al., 2004).

In this paper, we present a novel system based on CAN bus and provide evaluation experiments for the variable rate fertilizer application. The system structure consisting of the electro-hydraulic proportional valve control system, ground speed sampling ECU and light-bar guidance ECU is presented in this paper. The design of variable rate controller based on DSP and the closed loop control circuit based on proportional valve is discussed in details. Experimental results indicate that the control system can be used for variable rate fertilizer application based on CAN bus. Moreover, the system is open, easy to expand and communicate with other CAN bus based components. It also provides basis for other variable rate applications using CAN bus in precision agriculture.

\section{MATIERIALS AND METHOD}

\subsection{System Construction}

The control system links all units together via CAN bus. The variable rate controller based on DSP is the main node in charge of collecting signals from ground speed sampling unit and GPS receiver. The conveyor feed shaft rotate speed can be calculated using these signals and the prescription map stored in local memory. The variable rate fertilizer application is achieved by the PID control of the shaft speed. In the operation progress, the light-bar will help the operator to steer the machine moving along equal spacing parallel passes by lightening different number of LED. With the cooperation of these nodes, the variable rate application can be fulfilled successfully.

In our system, the electro hydraulic proportional valve is adopted as the control component (Figure 1). The developed variable rate controller compares the feedback signal from the optical rotary encoder mounted on the load shaft with an input demand to determine the speed error, and 
produces a PWM command signal to drive the electro hydraulic proportional valve. The control valve adjusts the flow of pressurized oil to move the hydraulic motor until the desired speed is attained.

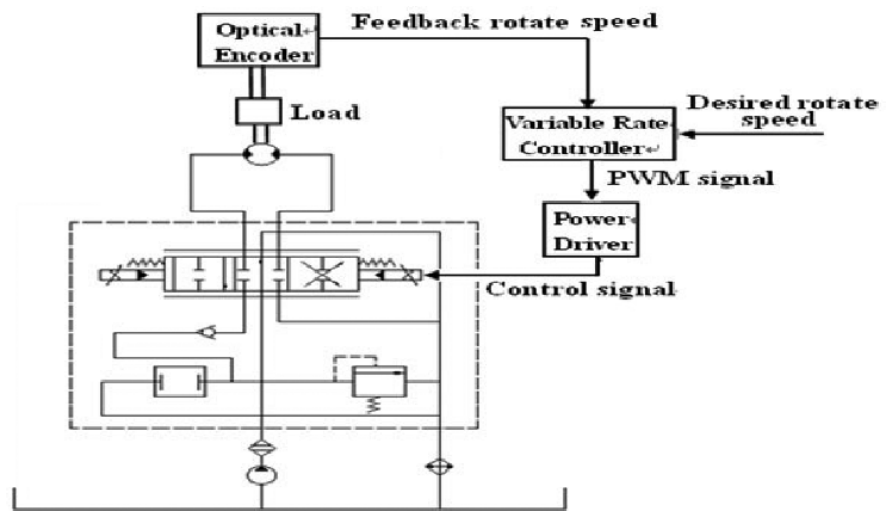

Figure 1. Block diagram of the hydraulic servo control system

\subsection{Design of Variable Rate Fertilizer Controller Based on DSP}

The variable rate fertilizer controller uses the TI TMS320LF2407A DSP as the main processor. The TMS320LF2407A provides several advanced peripherals such as 10-bit analog-to-digital converter (ADC), serial communications interface (SCI), PWM generation circuit and quadrature encoder pulse (QEP) circuit. The CAN controller has been integrated in the 2407A DSP, so it can realize the CAN communication just using a CAN transceiver $82 \mathrm{C} 250$.

In the fertilizer application, the PWM control signal for the electrohydraulic proportional valve is generated by the DSP PWM circuit. The QEP circuit decodes and counts the quadrature encoded input pulses from the optical rotary encoder as the feedback signal for the PID control. The LCD and keyboard are used for parameters input and system status display. The external FLASH memory is used for the storage of prescription map and guidance parallel lines information.

\section{EXPERIMENT}

In our system, the control object is the hydraulic motor and the feedback signal is the conveyor feed shaft rotate speed, and the output signal is the PWM pulses with changing duty cycles. If given a certain discharge volume of fertilizer $A r$ in one cell in the field and the machine's breadth $W i$, and 
the machine's moving speed from the sampling unit is Vt. Then the instantaneous flow rate of fertilizer $\mathrm{Fr}$ can be calculated by the following formula:

$$
\mathrm{Ar}=\mathrm{Fr} /(\mathrm{Vt} * \mathrm{Wi})
$$

where $A r$ is the fertilizer volume in prescription map, $F r$ is the instantaneous flow rate of fertilizer, $V t$ is the ground speed, $W i$ is the breadth of machine. The PID control is used here. The error between the desired rotate speed and the actual value measured by the optical rotary encoder is continuously sampling in a certain period for PID control.

A special protocol is design for the reliable communications. The protocol used 8-byte data as the basic unit of message frame. The 8 bytes data in the frame is arranged as follows:

1) The first byte is character ' $\$$ ', used as the start flag of frame.

2) The second byte is the type of the message, defined by ASCII code.

3) The last byte is character '\#', used as the end flag of frame.

4) The rest of bytes are used for carrying the parameters.

\section{CONCLUSION}

In this paper, a control system for variable rate application is presented. The hardware and software design of the control system are described in details. The evaluation experiment shows that the communication in the system using the developed protocol based on CAN bus is fast and reliable. The closed loop hydraulic servo control system is evaluated by the experiment through the PID control, which has a fast response and good performance. The development of this control system will make the variable rate application to implement easily and in a flexible way, and lead to the standardization of the electronic equipment in agricultural machinery.

\section{ACKNOWLEDGEMENTS}

Our project is supported by the Chinese National 863 Plan (Project No. 2006AA10A306).

\section{REFERENCES}

Maohua Wang. 1995. The Development of Precision Agriculture and Innovation of Engineering Technology. Transactions of the CSAE 15(1):1-8.

Marvin L. Stone and Kevin D. McKee. 1999. ISO 11783: An Electronic Communications Protocol for Agricultural Equipment, Agricultural Equipment Technology Conference, Louisville, Kentucky. 